\title{
Status of the NICA project at JINR
}

\author{
Vladimir Kekelidze ${ }^{1}$, Alexandr Kovalenko ${ }^{1, \star}$, Rihard Lednicky ${ }^{1}$, Viktor Matveev ${ }^{1}$, Igor Meshkov ${ }^{1}$, \\ Alexandr Sorin ${ }^{1}$, and Grigory Trubnikov ${ }^{1}$ \\ ${ }^{1}$ Joint Institute for Nuclear Research, Dubna, 141980 Russia
}

\begin{abstract}
The NICA (Nuclotron-based Ion Collider fAcility) project is now under active realization at the Joint Institute for Nuclear Research (JINR, Dubna). The main goal of the project is a study of hot and dense strongly interacting matter in heavy-ion (up to $\mathrm{Au}$ ) collisions at the center-of-mass energies up to $11 \mathrm{GeV}$ per nucleon. Two modes of operation are foreseen, collider mode and extracted beams, with two detectors: MPD and BM@N. The both experiments are in preparation stage. An average luminosity in the collider mode is expected to be $10^{27} \mathrm{~cm}^{-2} \mathrm{~s}^{-1}$ for $\mathrm{Au}(79+)$. Extracted beams of various nuclei with maximum momenta of $13 \mathrm{GeV} / \mathrm{c}$ (for protons) will be available. A study of spin physics with extracted and colliding beams of polarized deuterons and protons at energies up to $27 \mathrm{GeV}$ (for protons) is foreseen with the NICA facility. The proposed program allows one to search for possible signs of phase transitions and critical phenomena as well as to shed light on the problem of the nucleon spin structure.

Keywords: baryonic matter, heavy-ion interactions, fixed target and collider experiments, spin physics research
\end{abstract}

\section{Introduction}

The new research facility NICA aimed at study of heavy-ion and polarized proton and deuteron collisions is under design and construction at the Joint Institute for Nuclear Research (JINR) since 2010 [1]. The study of hot and dense baryonic matter should shed light on: in-medium properties of hadrons and the nuclear matter equation of state (EOS); the onset of deconfinement (OD) and/or chiral symmetry restoration (CSR); phase transition (PT), mixed phase and the critical end-point (CEP); possible local parity violation in strong interactions (LPV) [2-5]. It is indicated in a series of theoretical works, in particular, in [4] that heavy-ion collisions at $\sqrt{ } S_{N N} 11 \mathrm{GeV}$ allow attaining the highest baryon density. These calculations are illustrated in figure 1 . The NICA research domain is attractive as being the expected region for searching of new phenomena at the maximum baryon density including possible phase transitions. The study of the nucleon spin content ("spin puzzle") and polarization phenomena in light- and heavy-ion interactions is another target of research at NICA. The high intensity and high polarization $(>50 \%)$ of colliding beams could provide unique possibilities for this study.

The NICA construction plan up to 2023 foresees an essential development of the accelerator facility and construction of:

- the new spectrometer Baryonic Matter at Nuclotron (BM@N) in order to start the fixed target experiments with heavy ion beams extracted from the modernized Nuclotron;

\footnotetext{
^e-mail: kovalen@dubna.ru
} 

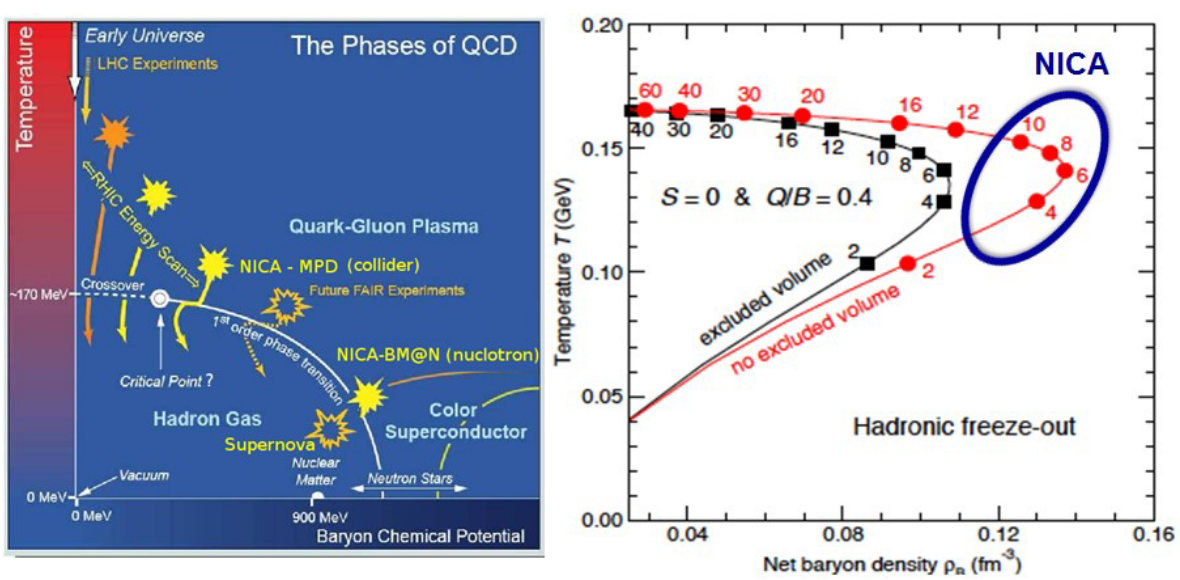

Figure 1. Freeze-out diagram for baryonic matter showing baryon density reachable at different facilities both in collider and fixed target experiments [4].

- the MultiPurpose Detector (MPD) at the NICA colliding beams with a primary goal to study heavy-ion collisions;

- preparation of the collider detector project "Spin Physics Detector" (SPD) aimed at a primary goal to study spin physics.

\section{The NICA accelerator complex progress}

The Nuclotron at Veksler and Baldin Laboratory of High Energy Physics (VBLHEP) of JINR was put into operation in 1993. It is based on the unique technology of superconducting fast cycling magnets developed at the Laboratory. The NICA facility (figure 2) includes: the injection complex, the booster, the upgraded Nuclotron and two storage rings with two interaction points IP1 and IP2 aimed at the MPD and SPD detectors respectively. The injection complex provides a wide set of ion species up to the heaviest one, $\mathrm{Au}$, at an energy of $3.5 \mathrm{MeV} / \mathrm{u}$ with an expected intensity of $2 \cdot 10^{9}$ particles per cycle. The main efforts during the last few years were devoted to completion of the design, manufacturing and commissioning beam of both heavy ion, KRION-6T, and polarized proton and deuteron, SPI, sources aimed at reaching of the specified parameters, manufacturing of the HILAC and the new RFQ fore-injector that should replace the old high voltage pulsed transformer at the linac LU-20. The design and manufacturing of the equipment were carried out in collaboration with German and Russian research laboratories and companies [6]. The HILAC was manufactured at Bevatech company (Frankfurt, Germany) in accordance with technical specification developed by the LHEP experts. Completion of the injector's manufacturing and its tests at the accelerator together with the ion sources were being performed in 2015-2016. The source of polarized ions (SPI) with the new RFQ pre-accelerating section was assembled at the linac LU-20 in May 2016. The beam was accelerated in LU-20 and some days later, 12 of June, polarized deuteron beam from SPI was accelerated in the Nuclotron ring (figure 3). All equipment of HILAC was also assembled and tested with a beam. The specified technical parameters were reached. The illustrations below show a general view of the HILAC. The unique RF power supply system based on solid state transistors was designed, manufactured and shipped to Dubna. The booster synchrotron should accelerate ions up to $600 \mathrm{MeV} / \mathrm{u}$. The magnetic ring of $211 \mathrm{~m}$ long is placed inside the window of the Synchrophasotron yoke. Technical 


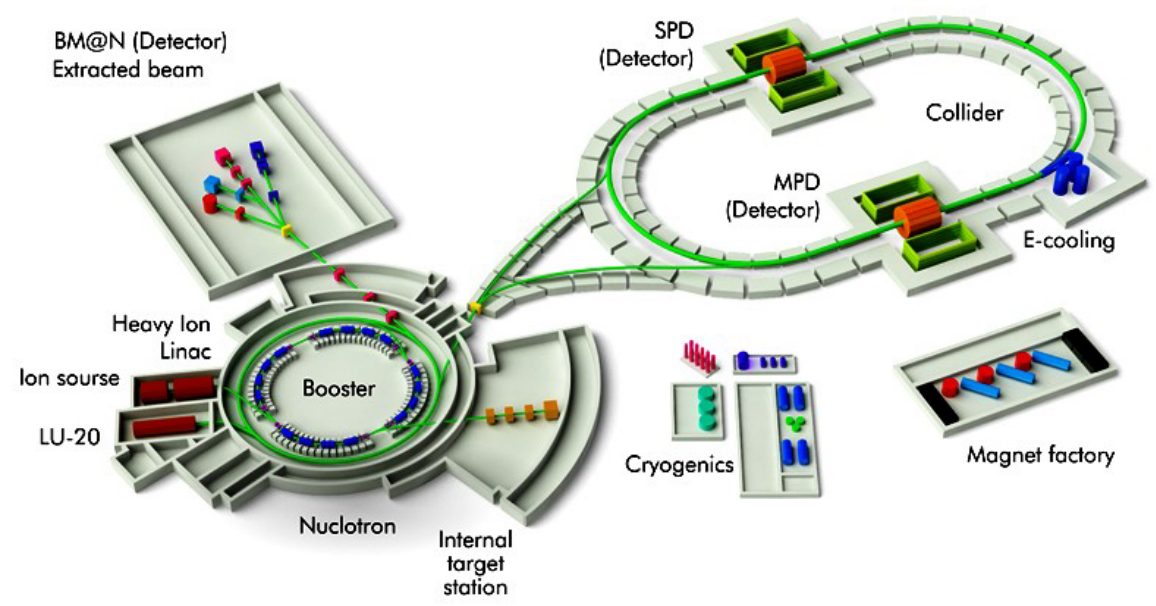

Figure 2. The NICA facility at the Laboratory of High Energy Physics of JINR.
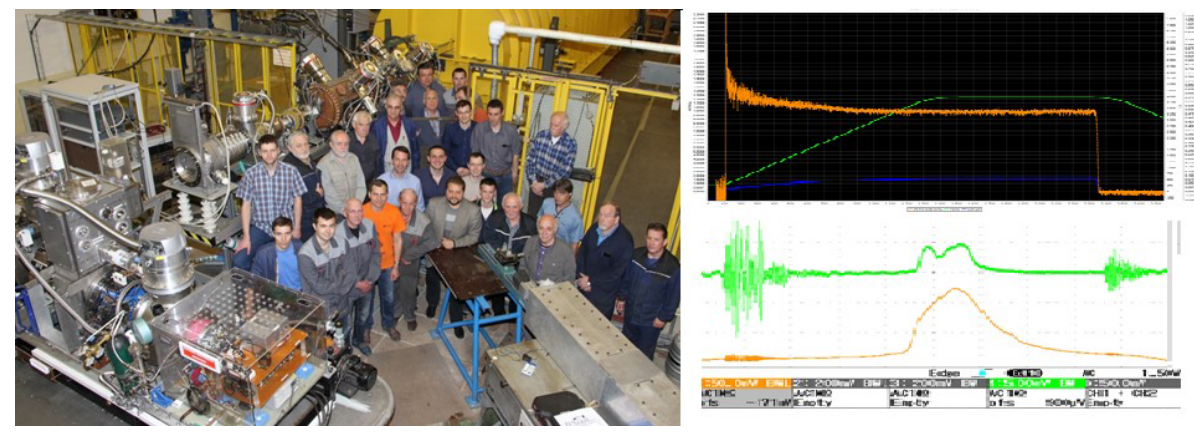

Figure 3. Basic equipment of the NICA polarized proton and deuteron fore-injector at the linac and the team.

design of the booster systems was performed. The most specific features of the booster are ultrahigh vacuum and the electron cooling system. The magnetic system elements were designed by the LHEP designers. The hardware parts like cryostats, basements etc. are under manufacturing at different factories out of Dubna. The most delicate part of the magnet production, namely: SC cable and coil fabrication, the cold mass assembling, magnetic measurements (warm and cold) and the bench tests are provided by the LHEP engineers and technicians at the Magnet Test Facility (figure 5). The serial fabrication and tests of the magnets for the NICA facility were started in 2016 [7].

The NICA cryogenics will be based on the modernized liquid helium plant that was built in the early 1990s for the Nuclotron. The main goals of the modernization are: increasing of the total refrigerator power from 4000 to $8000 \mathrm{~W}$ at $4.5 \mathrm{~K}$, making a new distribution system of liquid helium and ensuring the shortest possible cool-down time. These goals will be achieved by means of an additional $1000 \mathrm{l} / \mathrm{h}$ helium liquefier and satellite refrigerators located near the accelerator rings. A Nitrogen system will be used for magnet thermal shield cooling at $77 \mathrm{~K}$ and at the first stage of cooling down all three rings of the Nuclotron/NICA with the total length of about $1 \mathrm{~km}$ and cold mass of 290 tons. Work is in a good progress. 


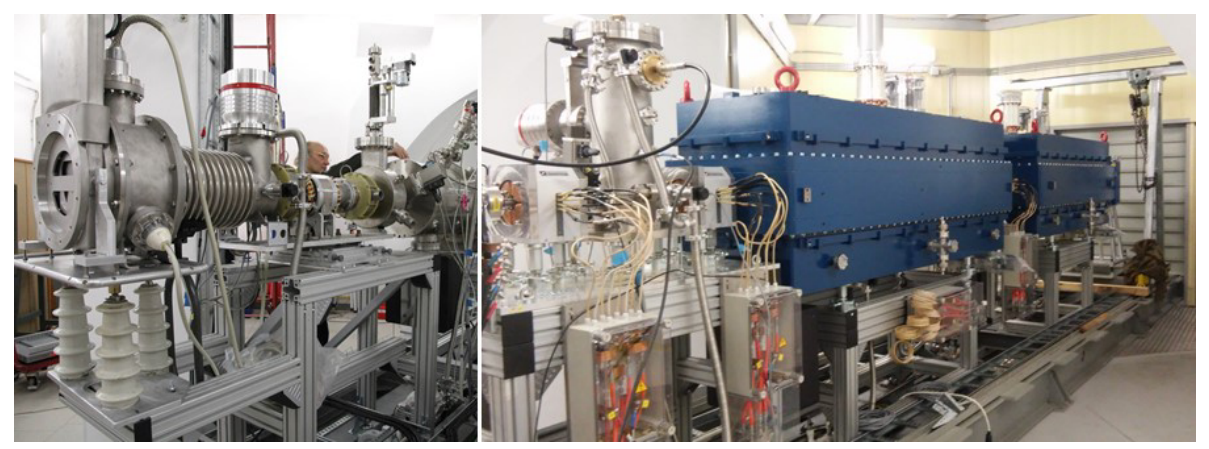

Figure 4. Main parts of HILAC: left - RFQ, right - buncher and two DTL accelerating sections.
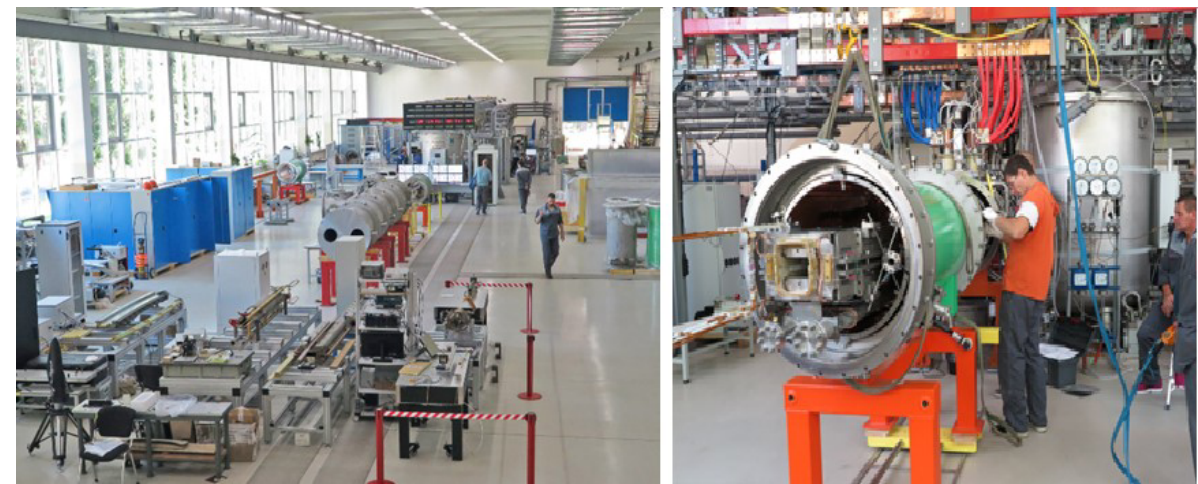

Figure 5. The NICA magnet test facility: left part - general view; right part - the booster dipole is being prepared for cold tests.

The two storage/collider rings will have two intersection points (IP). The major parameters of the NICA collider are the following: the maximum $\mathrm{B} \rho=45 \mathrm{Tm}$; vacuum in a beam chamber $-10-11$ Torr; maximum dipole field $2 \mathrm{~T}$; ion kinetic energy range from $1 \mathrm{GeV} / \mathrm{u}$ to $4.5 \mathrm{GeV} / \mathrm{u}$ for Au79+; zero beam crossing angle at IP; $9 \mathrm{~m}$ space for detector allocations at IP's; average luminosity L $=1027$ $\mathrm{cm}^{-2} \mathrm{~s}^{-1}$ for gold ion collisions at $\sqrt{ } \mathrm{s}_{N N}=9 \mathrm{GeV}$. The collider ring $503.04 \mathrm{~m}$ long (twice as large as the Nuclotron ring) has a racetrack shape and is based on double-aperture (top-to-bottom) superferric magnets - dipoles and quadrupoles. The TDR of the collider has been prepared, nevertheless final design of some lattice elements are still necessary. Current construction program considers the collider rings commissioning time consistent with the MPD construction.

\section{The BM@N experiment}

The main research goals of the new setup - BM@N (Baryonic Matter at Nuclotron) at extracted Nuclotron beams are the following

1. Study of the properties of dense nuclear (dominantly baryonic) matter with strangeness in heavy-ion $(\mathrm{A}+\mathrm{A})$ collisions in particular: 
- production mechanisms and modifications of hadron properties in dense nuclear matter ("inmedium effects") using different probes, namely: strange mesons, strange and multi-strange baryons; vector mesons via hadronic or dilepton/photon mode).

- study of the EoS with strangeness;

- hyper-matter production: search for light hypernuclei and multi-strange metastable objects.

2. Study of elementary reactions: pp, pn(d) as "reference" to pin down nuclear effects

3. Search for ,cold' nuclear matter with $\mathrm{pA}$ - collisions.

$\mathrm{BM} @ \mathrm{~N}$ at the first stage can study the in-medium effects on strangeness measuring of a variety of observables at different energies and centralities in heavy-ion collisions in order to find an "anomalous" behavior in comparison with theory. The observables sensitive to in-medium effects are the following: particle yields and ratios $\mathrm{p}_{T}-\left(\mathrm{m}_{T}\right)$-spectra, rapidity distributions, angular distributions, collective flow. The BM@N setup uses the existing wide aperture dipole magnet; a tracking system con-
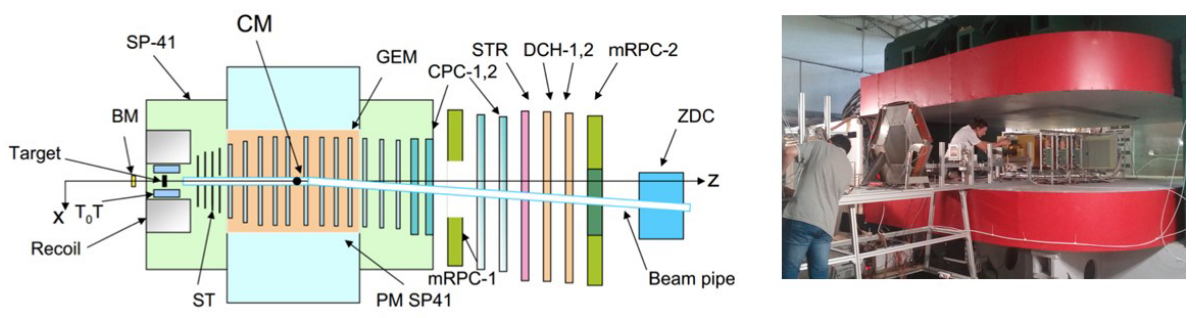

Figure 6. The BM@N scheme: GEM - central tracker inside analyzing magnet; DCH+Straw - outer tracker behind the magnet; $\mathrm{mRPC}+\mathrm{T} 0$ - time-of-flight system; ZDC - centrality measurements.

sisting of 12 planes of high resolution gem chambers (GEM) and 8 planes of drift chambers (DCH); a time-of-flight (TOF) system based on the RPC chambers and a fast counter detector providing trigger signal; a zero degree hadron calorimeter (ZDC) for the reaction plane definition and estimation of the impact parameter. The main goal for the coming period is to start physics data taking at the set-up
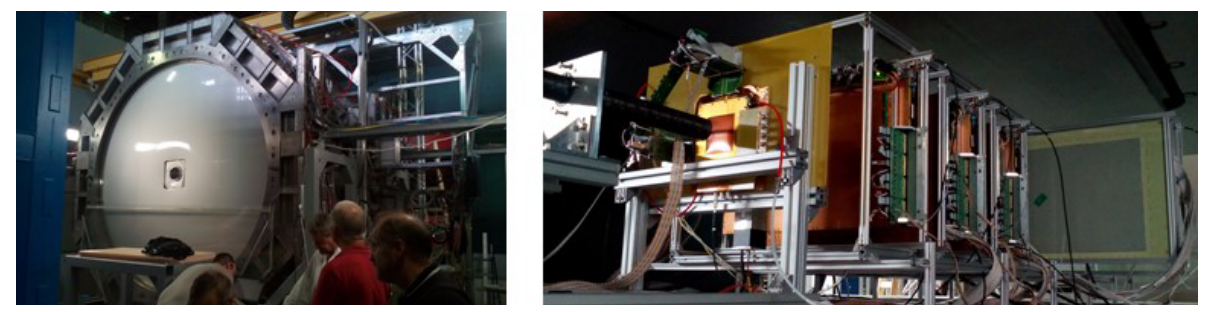

Figure 7. View of the assembled and tested BM@N detectors.

by the end of 2017. The further upgrade is foreseen at the second stage to equip the setup with a silicon vertex detector (in cooperation with GSI, Darmstadt), and with an electromagnetic calorimeter. The heavy-ion collision experiments at BM@N located in the fixed-target hall of the Nuclotron will provide a competitive research program focused on physics of dense nuclear and strange matter. 


\section{The MPD}

The MPD experimental program is aimed to investigate both hot and dense baryonic matter and polarization phenomena. Preliminary list of the first priority physics tasks to be performed includes:

measurement of a large variety of signals at systematically changing conditions of collision (energy, centrality, system size) using as bulk observables $4 \pi$ geometry particle yields (OD, EOS); multi-strange hyperon yields and spectra (OD, EOS); electromagnetic probes (CSR, OD); azimuthal charged-particle correlations (LPV); event-by-event fluctuation in hadron productions (CEP); correlations involving $\pi, \mathrm{K}, \mathrm{p}, \Lambda$ (OD); directed and elliptic flows for identified hadron species (EOS,OD); reference data (i.e. $p+p$ ) will be taken at the same experimental conditions. Technical design of the
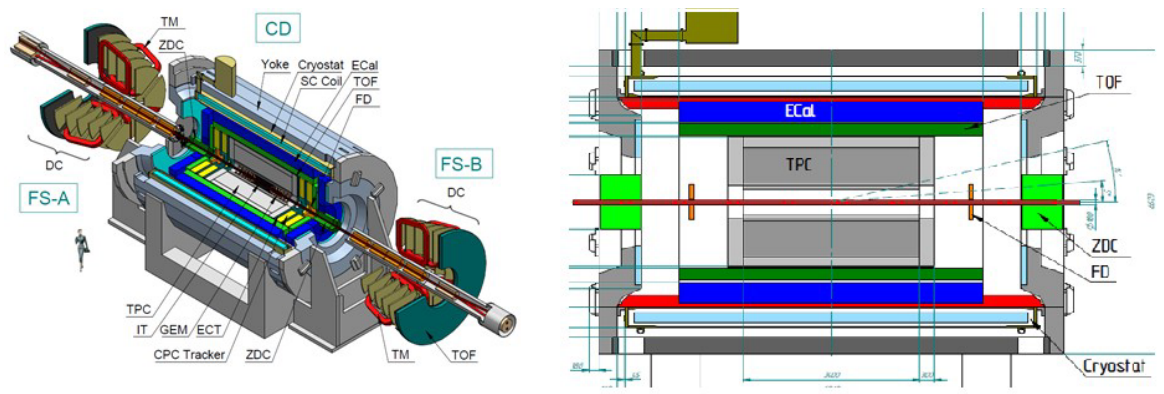

Figure 8. View of the MPD setup and the cross section of the central (barrel) part. The first stage detectors are: TPC, TOF, ECAL, ZDC, FD. Stage two includes IT and Endcaps.

detectors including beam tests is completed. The preparation for mass production is organized. Special problems, like the ECAL manufacturing and the design of this system with necessary capability for assembling inside the SC solenoid was also solved.

The status of MPD superconducting solenoid gives definite optimism: the contract with ASG company (Italy) was signed, works are in progress. The process of manufacturing heavy metallic parts of the solenoid yoke is illustrated at figure 9. The designed TPC assembly tools (see figure 10),
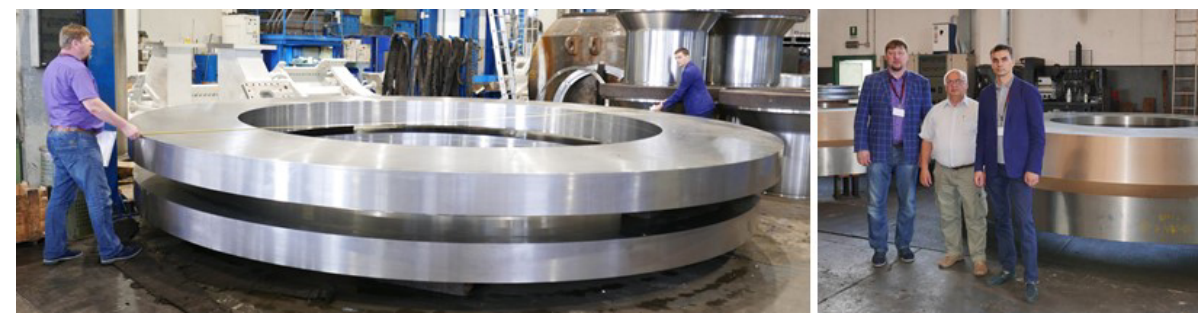

Figure 9. View of the solenoid yoke parts under manufacturing at the Vitkovice Heavy Machinery (CZ).

cooling and laser calibration system provides minimal TPC deformation $(\sim 32 \mu \mathrm{m})$ and is ready for production. The processes studied with the MPD were simulated using the dedicated software framework (MpdRoot). This software is based on the object - oriented framework FairRoot [8] and provides a powerful tool for detector performance studies, development of algorithms for reconstruction and physics analysis of the data. Based on the modeling, the MPD physics cases at the first stage (2023) are shown in table 1 below. 


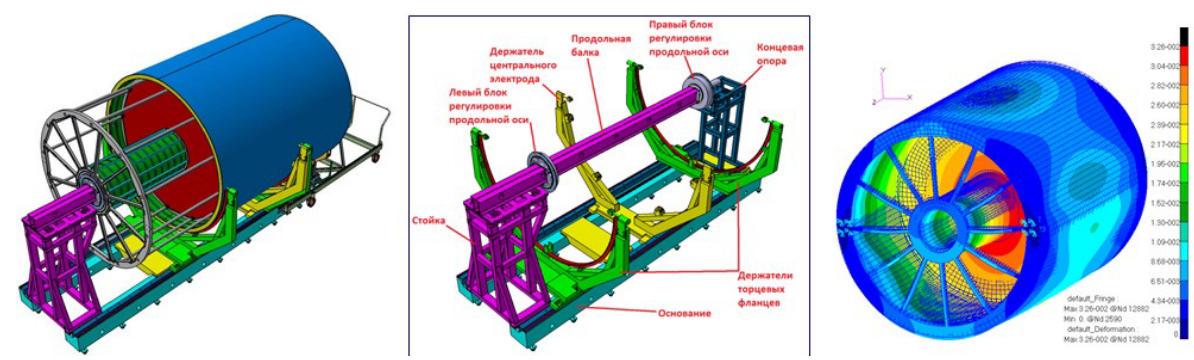

Figure 10. The TPC assembly tools.

Table 1. MPD physics cases at the first stage (2023).

\begin{tabular}{|c|c|c|c|}
\hline Observable & Set-up & Coverage & New insights \\
\hline $\begin{array}{l}\text { Hadron yields \& } \\
\text { ratios }\end{array}$ & $\begin{array}{l}\text { TPC, TOF, } \\
\text { FHCAL,ECAL }\end{array}$ & $\begin{array}{l}|\eta|<1.5 \\
\text { pT }<4 \mathrm{GeV} / \mathrm{c}\end{array}$ & $\begin{array}{l}\text { Data for } 4<\sqrt{ }<<7 \mathrm{GeV} \text {, critical } \\
\text { assessment of } y \text {-spectra and } K / \pi \text {-ratio }\end{array}$ \\
\hline $\begin{array}{l}\text { Hyperons: } \\
\text { yields, flow, } \\
\text { Polarization }\end{array}$ & $\begin{array}{l}\text { TPC, TOF } \\
\text { FHCAL }\end{array}$ & $\begin{array}{l}|\eta|<1.5 \\
\mathrm{pT}<4 \mathrm{GeV} / \mathrm{c}\end{array}$ & $\begin{array}{l}\text { New data on yields, flow and } \\
\text { polarization at } \sqrt{ } s<7 \mathrm{GeV} \text {. }\end{array}$ \\
\hline Dileptons & $\begin{array}{l}\text { TPC, TOF } \\
\text { ECAL, FHCAL }\end{array}$ & $\begin{array}{l}|\eta|<1.2 \\
\text { pT }<3 \mathrm{GeV} / \mathrm{c}\end{array}$ & low statistics data for comparison \\
\hline $\begin{array}{l}\text { Fluctuations \& } \\
\text { Correlations }\end{array}$ & $\begin{array}{l}\text { TPC, TOF } \\
\text { ECAL, FHCAL }\end{array}$ & $|\eta|<1.5$ & $\begin{array}{l}\text { New data on Ev-by-Ev fluct. } \\
\text { for } \sqrt{s}>4 \mathrm{GeV}\end{array}$ \\
\hline $\begin{array}{l}\text { Chiral Magnetic } \\
\& \text { vortical effects }\end{array}$ & $\begin{array}{l}\text { TPC, TOF } \\
\text { FHCAL }\end{array}$ & $\begin{array}{l}|\eta|<1.5 \\
\mathrm{pT}<3 \mathrm{GeV} / \mathrm{c}\end{array}$ & $\begin{array}{l}\text { Data @ } \sqrt{\mathrm{s}}<7 \mathrm{GeV}(\mathrm{CME}) \\
\text { Vortical @ } 4<\sqrt{\mathrm{s}}<11 \mathrm{GeV}\end{array}$ \\
\hline (Hyper)Nuclei & $\begin{array}{l}\text { TPC, TOF } \\
\text { ZDC }\end{array}$ & $\begin{array}{l}|\eta|<1.5 \\
\mathrm{pT}<5 \mathrm{GeV} / \mathrm{c}\end{array}$ & low statistics data for comparison \\
\hline
\end{tabular}

This stage is characterized by limited luminosity (to $5 \cdot 10^{25} \mathrm{~cm}^{-2} \mathrm{~s}^{-1}$ ). The evaluated rate in $\mathrm{Au}$ $+\mathrm{Au}$ collisions at the maximum energy (10\% central interactions) will be up to $7 \mathrm{kHz}$ taking into account the designed luminosity of $\mathrm{L}=1 \cdot 10^{27} \mathrm{~cm}^{-2} \mathrm{~s}^{-1}$. Groups from 12 institutions are working on the sub-detector prototyping and serial productions. More detailed information could be found in the conceptual design report [9] or at the NICA site: " nica.jinr.ru ". It was confirmed that the MPD is well optimized for studying in-medium effects caused by high baryon densities, such as: changing particle properties in hot and dense medium (broadening spectral functions etc.), event-byevent dynamical fluctuations of strange to non-strange particle ratios and others. There are optimal conditions at both BM@N and MPD detectors for observation and study of light hypernuclei. The simulations demonstrate enhanced yields of different single and double $\Lambda$ light hydrogen and helium nuclei as a function of $\sqrt{ } \mathrm{s}_{N N}$. There are some novel proposals such as studying vorticity [10], helicity separation in heavy-ion collisions [11], and directed flow in asymmetric nuclear collisions [12] that could be studied at NICA at stage two.

The tests of the detector prototypes have been carried out at the Nuclotron test beams in several past years and will be continued in 2017 and after. 


\section{Status of the SPD preparation}

The NICA program foresees that the SPD detector will be designed and installed at the IP-2 to study spin physics. The comparable parameters on measurements of different components of transverse quark-parton distribution functions are presented below. There is a number of processes besides the

Table 2. The comparable parameters on measurements of different components of transverse quark-parton distribution functions.

\begin{tabular}{|c|c|c|c|c|c|c|}
\hline experiment & $\begin{array}{l}\text { CERN, } \\
\text { COMPASS-II }\end{array}$ & $\begin{array}{l}\text { FAIR, } \\
\text { PANDA }\end{array}$ & $\begin{array}{r}\text { FNAL, } \\
\text { E-906 }\end{array}$ & $\begin{array}{l}\text { RHIC, } \\
\text { STAR }\end{array}$ & $\begin{array}{l}\text { RHIC- } \\
\text { PHENIX }\end{array}$ & $\begin{array}{l}\text { NICA, } \\
\text { SPD }\end{array}$ \\
\hline mode & F.T. & F.T. & F.T. & collider & collider & collider \\
\hline Beam/target & $\pi-, \quad p$ & anti-p, p & $\pi-, p$ & pp & pp & $p p, p d, d d$ \\
\hline Polarization:b/t & $0 ; \quad 0.8$ & 0 & $0 ; \quad 0$ & 0.5 & 0.5 & 0.7 \\
\hline Luminosity & $2 \cdot 10^{33}$ & $2 \cdot 10^{32}$ & $3.5 \cdot 10^{35}$ & $5 \cdot 10^{31}$ & $5 \cdot 10^{31}$ & $10^{32}$ \\
\hline$\sqrt{s}, \mathrm{GeV}$ & 14 & 6 & 16 & 200,500 & 200,500 & $10-26$ \\
\hline$x_{1 \text { (beam) }}$ range & $0.1-0.9$ & $0.1-0.6$ & $0.1-0.5$ & $0.03-1.0$ & $0.03-1.0$ & $0.1-0.8$ \\
\hline$q_{T}, \mathrm{GeV}$ & $0.5-4.0$ & $0.5-1.5$ & $0.5-3.0$ & $1.0-10.0$ & $1.0-10.0$ & $0.5-6.0$ \\
\hline Lepton pairs, & $\mu-\mu+$ & $\mu-\mu+$ & $\mu-\mu+$ & $\mu-\mu+$ & $\mu-\mu+$ & $\mu-\mu+, e+e-$ \\
\hline Data taking & 2015 & $>2025$ & 2013 & $>2016$ & $>2016$ & $>2020$ \\
\hline Transversity & NO & NO & No & YES & YES & YES \\
\hline Boer-Mulders & YES & YES & YES & YES & YES & YES \\
\hline Sivers & YES & YES & YES & YES & YES & YES \\
\hline Pretzelosity & No & No & No & No & YES & YES \\
\hline Worm Gear & NO & NO & NO & No & NO & YES \\
\hline Direct $y$ & No & No & No & YES & YES & YES \\
\hline
\end{tabular}

mentioned, which could be studied with this detector and with the fixed target detectors at beams extracted from the upgraded Nuclotron, namely: DY processes with longitudinally and transversally polarized $\mathrm{p}$ and $\mathrm{d}$ beams; extraction of unknown (poorly known) parton distribution functions (PDF); PDFs from $\mathrm{J} / \psi$ production processes; spin effects in various exclusive and inclusive reactions; cross sections of diffractive processes; helicity amplitudes and double spin asymmetries (Krisch effect) in elastic reactions; spectroscopy of quarkoniums with any available decay modes. This can be done in the kinematic energy region which is not available at other experiments. The formation of the collaboration has been started. The analysis of the accelerator issues related to polarized proton and deuteron beams has been progressing during the last three years. Feasible schemes of the polarization control in the Nuclotron and collider have been proposed and analyzed [13, 14].

\section{Status of the NICA site preparation}

Preparation of the site for the collider and the detectors was started in 2013. The technical design project of the NICA collider building, the MPD and SPD halls and engineering infrastructure with the set of technical documentation was completed in 2014. The State expertise review has been successfully passed in accordance with the Russian regulations. A general contractor for civil construction of the NICA Complex is STRABAG Company. View of the site at the Conference date are shown in figure 11 . 


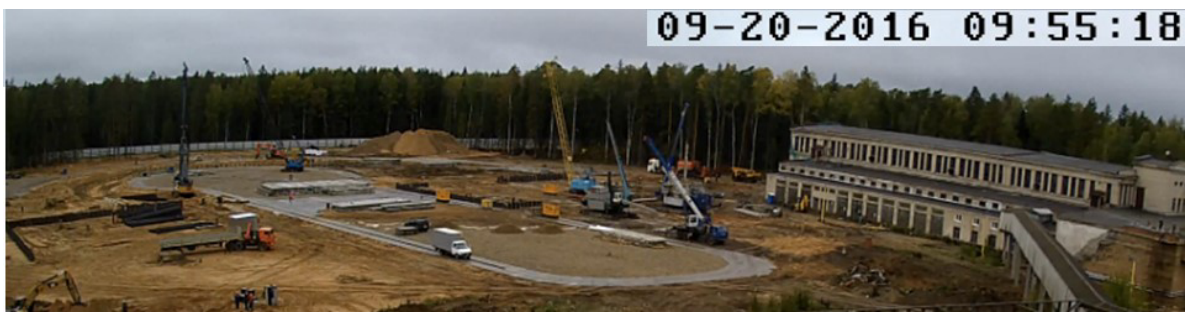

Figure 11. View of the NICA facility site in October 2016.

\section{Concluding remarks}

The NICA accelerator and experimental complex and the corresponding research program will provide a relevant research, which is both competitive and complementary to ones being carried out at other world leading scientific centres and facilities. The Agreement between the Government of the Russian Federation and the Joint Institute for Nuclear Research has been signed in June 2016. Additional special funds were allocated for the NICA Complex. Talking about NICA at the Academician A.M. Baldin Memorial Seminar, we would like to remind about the proposal of the universal collider design and construction - The Super-Nuclotron - the first collider project at JINR [15].

\section{References}

[1] V.D. Kekelidze et al., PoS (ICHEP2012) 411, (2013)

[2] A. Sissakian et al., Part. \& Nucl Lett. 5, 1 (2008)

[3] M. Gazdzicki et al., PL. B 585, 115 (2004)

[4] J. Cleymans, and J. Randrup, Phys. Rev. C 74, 04791 (2006)

[5] B. Mohanty, J. Phys. G: Nucl. \& Part. Phys. 38, 124023 (2011)

[6] A. Butenko et al., IPAC 2014, paper WEPRO067 (Dresden, June 2014)

[7] G. Trubnikov et al., ibid, paper TUPRO005 (Dresden, June 2014)

[8] D. Bertini et al., JoP,CS 119, 032011, (2008). http://cbmroot.gsi.de

[9] MPD CDR v.1.2 (JINR, Dubna, 2010). http://www.nica.ru

[10] O. Rogachevsky et al., PR C 82, 054910 (2010)

[11] M. Baznat et al., PR C 88, 061901 (2013)

[12] V.D. Toneev, private communication 2014

[13] A.D. Kovalenko et al., Part. \& Nucl 45(1) (2014)

[14] I.A. Savin et al., arXiv:1408.3959 [hep-ex], 2014

[15] A.M. Baldin, A.D. Kovalenko, JINR Rapid Comm. JINR - 3(77)-96, Dubna 1996 